\title{
Chromonic nematic phase and scalar order parameter of indanthrone derivative with ionic additives
}

\author{
Boiko O.P. ${ }^{1}$, Vasyuta R.M. ${ }^{1}$, Semenyshyn O.M. ${ }^{1}$, \\ Nastishin Yu.A. ${ }^{2}$ and Nazarenko V.G. ${ }^{1}$ \\ ${ }^{1}$ Institute of Physics, NAS of Ukraine, Kyiv, Ukraine \\ ${ }^{2}$ Institute of Physical Optics, 23 Dragomanov St., 79005 Lviv, Ukraine
}

Received: 08.10.2008

\begin{abstract}
We investigate influence of different ionic additives on the phase behaviour and scalar order parameter of lyotropic chromonic nematic liquid crystals formed by the molecules representing derivatives of indanthrone. $\mathrm{KI},\left(\mathrm{NH}_{4}\right)_{2} \mathrm{SO}_{4}$ and $\mathrm{NaCl}$ salts increase biphasic nematic region on the temperature-concentration phase diagram, whereas the scalar orientational order parameter is hardly sensitive to their presence. We suggest that these changes are attributed to increase in the aggregate length and polydispersity of the latter, while the interaction between aggregates remains unaffected. The $\mathrm{Na}_{2} \mathrm{SO}_{4}$ salt lowers anomalously the measured value of the scalar order parameter to 0.42 , for which the nematic phase should not exist. We address this anomaly to modification of the shape of chromonic aggregates due to growing of side chains within the rod-like aggregates. The $\mathrm{NH}_{4} \mathrm{Cl}$ salt does not affect the phase diagram and the scalar order parameter of Blue 250 chromonematic.
\end{abstract}

PACS: 78.20.Ci, 87.15.Mi, 78.20.Fm, 42.79.Kr, 61.30.Gd.

UDC: 535.34

Keywords: absorption spectra, dichroism, liquid crystals, chromonic nematics, scalar order parameter

\section{Introduction}

Liquid crystal (LC) materials being a key element of liquid crystal displays (LCDs) are present in LC panels in a form of thermotropic nematic of a low molecular weight, whose optical properties are controlled by external electric field. However, functional role of LCs in the LCDs has recently been expanded to solve different problems related to the quality of displayed images, using a new type of LCs, Lyotropic Chromonic Liquid Crystals (LCLC). Due to their high orientational ordering, negative optical birefringence and dichroism, LCLCs have been proposed as potential materials for internal retardation films, including viewing angle compensators and temperature compensating retardation films, optical rotators, polarizers and alignment layers incorporated into LC panels [1-6]. In water LCLC molecules associate into nano-sized aggregates, which then self-assemble 
into structures with long-range orientational order, i.e. into LCs [7-16]. Moreover, it has been shown that the long-range orientational order is preserved in dried chromonic films, when those films are deposited onto solid substrates from their LC-nematic state uniformly aligned by shear. Application of isotropic or unaligned LCLC solutions does not result in a homogeneously aligned optically anisotropic solidified film. Therefore, characterization of chromonic lyotropic nematic LCs and optimization of their physical properties are greatly demanded for reliable, high-performance dried films. Recent experiments [17] have demonstrated that the structure and physical properties of LCLCs are highly sensitive to the presence of doped ions in the solution. In this paper, we study systematically the phase behaviour and the order parameter for nematic LCLC solution as functions of ionic dopants added into the solution.

\section{Materials}

We studied a water-soluble LCLC formed by the material, which is a derivative of indanthrone called as Blue 250. The individual molecules have a plank-like shape and assemble into rod-like aggregates that form a nematic phase at room temperature once the concentration exceeds $4.5 \mathrm{wt} \%$. In water solution the molecules are in dissociated state. On the whole there are three cations per molecule on average, i.e. the average charge per molecule is -3 in the solution. The molecular structure of Blue 250 is the same as that of Blue 27 [12]; the only difference is a larger number of $\mathrm{NH}_{4}{ }^{+}$cations and the corresponding $\mathrm{SO}_{3}{ }^{-}$groups at the periphery of the molecule. Fig. 1 shows the chemical structure mentioned above in the dissociated state (the ammonium cations $\mathrm{NH}_{4}$ and the corresponding $\mathrm{SO}_{3}{ }^{-}$groups are omitted in Fig. 1).<smiles>O=C1C2=C(C(=O)C3C=CC=CC13)C1NC3=C(NC1C(Cl)=C2)C1C(=O)c2ccccc2C(=O)C1C=C3Cl</smiles>

Fig. 1. Chemical structure of Blue 250 in the dissociated state.

We used the ionic solutes of KI, LiI, NaI, $\mathrm{NaCl}, \mathrm{CaCl}_{2}, \mathrm{NH}_{4} \mathrm{Cl}$, $\left(\mathrm{NH}_{4}\right)_{2} \mathrm{SO}_{4}, \mathrm{Na}_{2} \mathrm{SO}_{4}$ and $\left(\mathrm{C}_{4} \mathrm{H}_{9}\right)_{4} \mathrm{NI}$, all of which are purchased from Sigma. The salts were added into the solution of Blue 250 dissolved in deionized water. Then the test-tubes with the solutions were stirred and held in ultrasonic water bath for $1 \mathrm{~h}$ at the temperature of about $10^{\circ} \mathrm{C}$ higher than the clearing point for complete dissolving of the salts. For all the solutions with the salt additives we used the concentration $\mathrm{c}=5.2 \mathrm{wt} \%$ of Blue 250 , which is equal to $0.074 \mathrm{M}$. 
In approximately $24 \mathrm{~h}$ after preparation, the solutions were filled into glass cells and sealed with epoxy glue. The cells were observed in polarizing microscope on heating (the rate $0.2^{\circ} \mathrm{C} / \mathrm{min}$ ) in order to identify the phase state of the solutions.

\section{Phase Behaviour}

The phase diagram of aqueous solutions of Blue 250 without additives is shown in Fig.2. The accuracy for determination of transition temperature was $\pm 0.5^{\circ} \mathrm{C}$ and the concentration was controlled with the accuracy better than $\pm 0.1 \%$. At the room temperature $\left(23^{\circ} \mathrm{C}\right)$ the aqueous solution of Blue 250 is in the isotropic $(I)$ phase for the weight concentrations of Blue 250 less than $4.5 \mathrm{wt} \%$. The results presented below are obtained for the concentration $\mathrm{c}=5.2 \mathrm{wt} \%(0.074 \mathrm{M})$, which demonstrates stable nematic phase on heating up to $35^{\circ} \mathrm{C}$. The phase diagram has relatively wide $\left(5^{\circ} \mathrm{C}\right)$ biphasic regions of coexisting $N$ and $I$ phases, which is typical for the other types of LCLC.

In agreement with Refs. [7-10, 18-23], samples with higher LCLC concentrations display higher temperatures for the nematic-to-isotropic transition. Physical properties of LCLCs and the phase transition temperatures are expected to be dependent on ionic content of the solution. Ionic additives might modify both the intra- (interactions between the molecules belonging to the same aggregate) and inter-columnar interactions, thus altering electrostatic forces between the charged groups at the lateral surfaces of aggregates [10, 24-26].

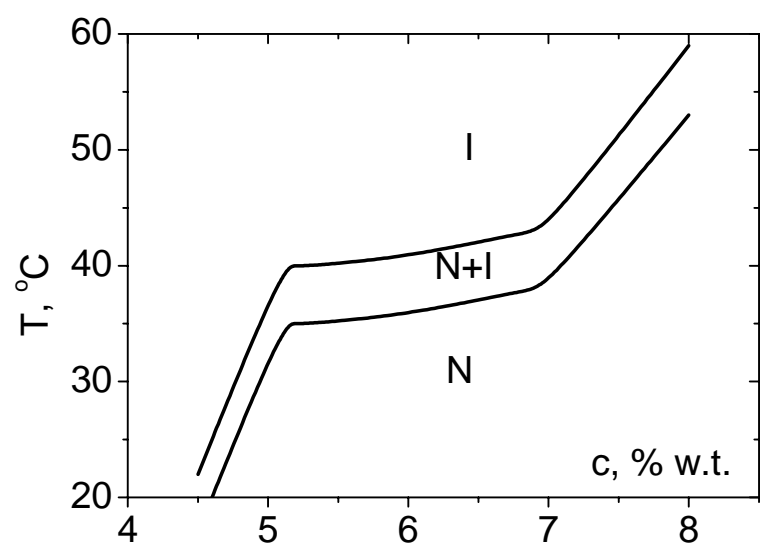

Fig. 2. Phase diagram for Blue 250/water composition.

For the concentrations under study (between $10^{-6} \mathrm{M}$ and $10^{-1} \mathrm{M}$ ) all the salts listed above are well soluble in Blue 250 water solution, except for $\mathrm{CaCl}_{2}$, though it remains soluble in water $\left(74 \mathrm{~g}\right.$ of $\mathrm{CaCl}_{2}$ per $100 \mathrm{~g}$ of water). The phase diagrams of the salt doped with aqueous solutions of Blue 250 are shown in Fig. 3.

We conclude that the salt additives studied by us can be divided into three groups. The first is represented by the salts $\mathrm{KI},\left(\mathrm{NH}_{4}\right)_{2} \mathrm{SO}_{4}$ and $\mathrm{NaCl}$, for which the biphasic temperature region $\Delta \mathrm{T}_{\mathrm{NI}}$ of the coexistence of nematic and isotropic phases increases with increasing salt concentration. The temperature range of the biphasic region is defined as $\Delta \mathrm{T}_{\mathrm{NI}}=\mathrm{T}_{\mathrm{I}}-\mathrm{T}_{\mathrm{N}}$, where $\mathrm{T}_{\mathrm{N}}$ and $\mathrm{T}_{\mathrm{I}}$ are the temperatures at which the first droplet of the iso- 

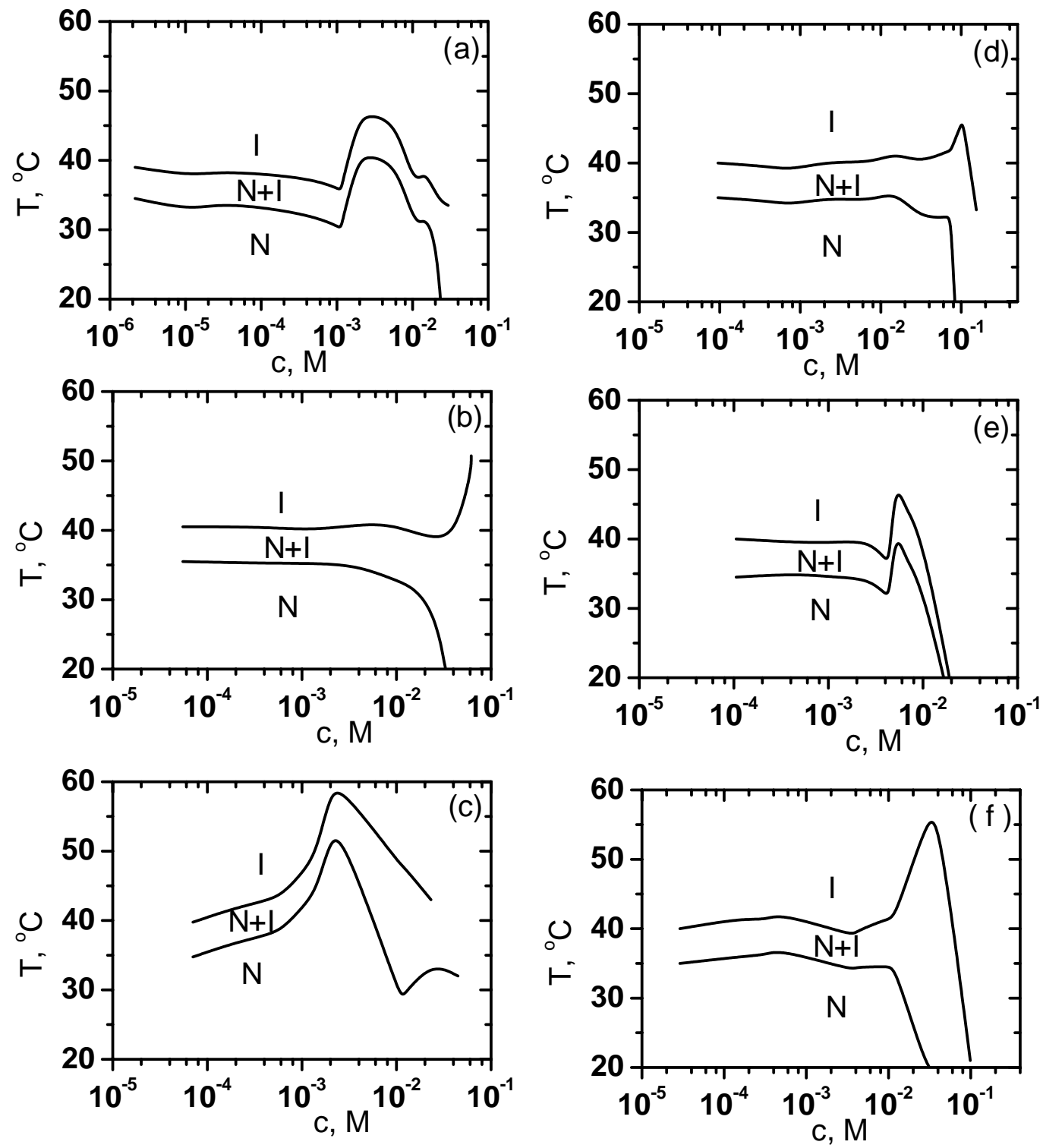

Fig. 3. Phase diagram of Blue $2505.2 \%$ wt with the ionic additives:
(a) $\left(\mathrm{CH}_{3}\right)_{4} \mathrm{NI}$,
(b) $\mathrm{KI}$,
(c) Lil,
(d) $\mathrm{NaCl}$,
(e) $\mathrm{Na}_{2} \mathrm{SO}_{4}$ and
(f) $\left(\mathrm{NH}_{4}\right)_{2} \mathrm{SO}_{4}$

tropic phase appears and, correspondingly, the last nematic droplet disappears on heating. For these salts $\Delta \mathrm{T}_{\mathrm{NI}}$ can be as large as $25^{\circ} \mathrm{C} . \Delta \mathrm{T}_{\mathrm{NI}}$ increases due to increasing $\mathrm{T}_{\mathrm{I}}$, accompanied by a decrease of $\mathrm{T}_{\mathrm{N}}$. Taking into account that for the salt-free Blue 250 solutions the parameter $\Delta \mathrm{T}_{\mathrm{NI}}$ remains constant (approximately $5^{\circ} \mathrm{C}$ for a wide range of dye concentrations), we can relate the salt-induced broadening of the biphasic temperature region to enhancement of polydispersity of the aggregates [27, 28].

We did not observe broadening of the biphasic region for the additives of the second group represented by $\left(\mathrm{C}_{4} \mathrm{H}_{9}\right)_{4} \mathrm{NI}$ and $\mathrm{Na}_{2} \mathrm{SO}_{4}$. In a wide concentration region both $\mathrm{T}_{\mathrm{N}}$ and $\mathrm{T}_{\mathrm{I}}$ increase when the salt concentrations do. Finally, $\mathrm{NH}_{4} \mathrm{Cl}$ belongs to the third group for 
which addition of the salt does not induce any noticeable changes in the phase diagram of the solution of Blue 250 .

When the molar fractions of the salt and LCLC become comparable (around $0.07 \mathrm{M}$ ), all the solutions transform into isotropic state for the temperatures above $20^{\circ} \mathrm{C}$. Visual observations indicate that viscosity of the solutions increases with increasing salt concentration and the solutions become quite viscous even in the isotropic phase at high enough salt concentrations.

\section{Spectroscopic measurements}

To shed light on the nature of the salt-induced modifications observed on the phase diagram of the dye Blue 250, we have measured optical absorption spectra of the solutions under test. The idea of our experiments is to verify whether the spectra and, thus, the structure of the chromonic aggregates of the salt-doped solution are sensitive to presence of the salt. In addition, one can directly deduce scalar orientation order parameter from the absorption spectral data for the aligned LCLC samples.

Spectroscopic measurements were performed on the aligned samples of each solution of Blue 250 and with the corresponding additives. In this work, we used spin-coated and buffed films of polyimide SE-7511 (Nissan Chemical Industries, Ltd., Japan) to align the $N$ phase of LCLCs in a planar fashion, along a single direction in the plane of bounding substrate determined by the direction of buffing. Glass substrates were washed in an ultrasonic bath of Alconox detergent and water for $10 \mathrm{~min}$ at $60^{\circ} \mathrm{C}$ and then removed and dried. The polyimide SE-7511 Sunever (grade 7511L) and the solvent Sunever (both from Nissan Chemical Industries, Ltd., Japan) were mixed in the proportion 1:1. The solution was coated onto the glass substrates by a spin-coater at $1500 \mathrm{rpm}$. The polymer layer was dried at $100^{\circ} \mathrm{C}$ for $2 \mathrm{~min}$ and then baked at $180^{\circ} \mathrm{C}$ for $1 \mathrm{~h}$. The polyimide-coated substrates were rubbed using an aluminium block covered with felt. The cells were assembled from pairs of substrates, with the rubbing directions being anti-parallel to each other.

The thickness of the gap between the glass plates was measured with interference technique for the empty cells. The cells were filled at the temperature about $10^{\circ} \mathrm{C}$ higher than the clearing point by a capillary flow assisted by negative pressure, and then promptly sealed. The flow direction and the pressure gradient were parallel to the buffing direction of the polyimide layers. The samples were also heated to about $10^{\circ} \mathrm{C}$ above the isotropic transition prior to each measurement, in order to mitigate prehistory effects mentioned in the work [10]. The measurements were performed $24 \mathrm{~h}$ after cooling down to the $N$ phase.

The absorption spectra were measured on the aligned samples of LCLCs, using Perkin Elmer Lambda 35 UV-VIS-IR spectrophotometer. The sample was placed between a pair of parallel polarizers. To exclude the effects of reflection at the cell interfaces, the transmission was measured with respect to the light transmitted through the identical cell filled with water, instead of LCLC. 
All of the measured spectra of Blue 250 for the studied solution with the ionic additives exhibit the same form and position of the absorption band. The changes (shifting or reshaping of the absorption band) if any are very small. The main spectral changes reflect the changes associated with the orientational distribution of the absorption dipoles, which can be characterized in terms of orientation scalar order parameter. Fig. 4 represents the absorption spectra for the polarizations parallel ( II ) and perpendicular $(\perp)$ to the director orientation, which are normalized to the corresponding absorption maximum of the pure Blue 250 dye and those with the ionic additives KI, NaI and $\left(\mathrm{C}_{4} \mathrm{H}_{9}\right)_{4} \mathrm{NI}$. The concentration for all the additives is $10^{-2} \mathrm{M}$.

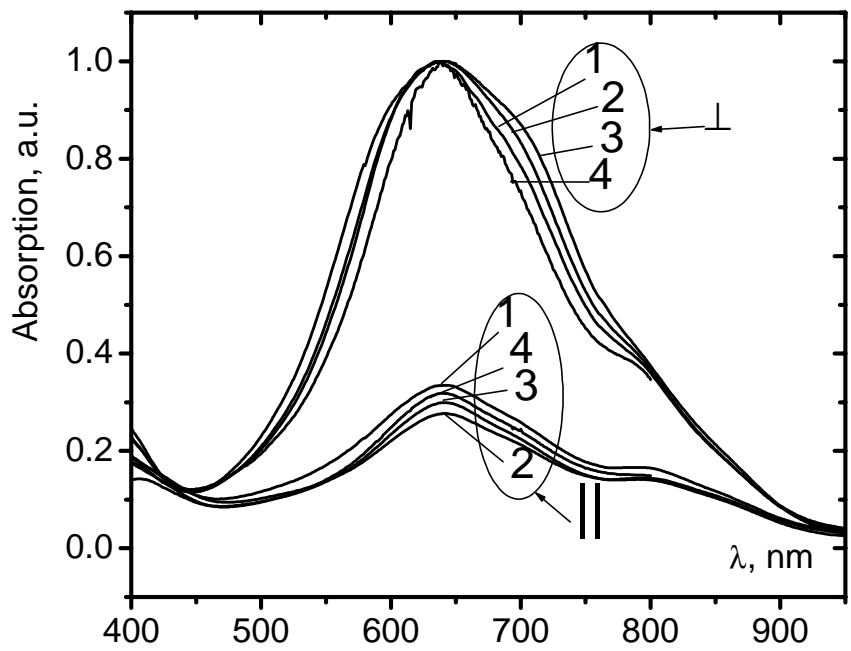

Fig. 4. Absorption spectra obtained for the light polarized along (II) and perpendicular $(\perp)$ to the director orientation for Blue $250 \quad 5.2 \mathrm{wt} \%$ with the corresponding ionic additives: (1) pure Blue 250, (2) $\left(\mathrm{C}_{4} \mathrm{H}_{9}\right)_{4} \mathrm{NI}$ (3) KI and (4) Nal.

The increase in the salt concentration results in slight decrease of $\mathrm{N}=\mathrm{k}_{\|} / \mathrm{k}_{\perp}$, when compare to the pure Blue 250 solution. The only exception is $\mathrm{Na}_{2} \mathrm{SO}_{4}$. Sodium sulfate shows significant decrease in the absorption anisotropy of LCLC, which is clearly seen from Fig. 5.

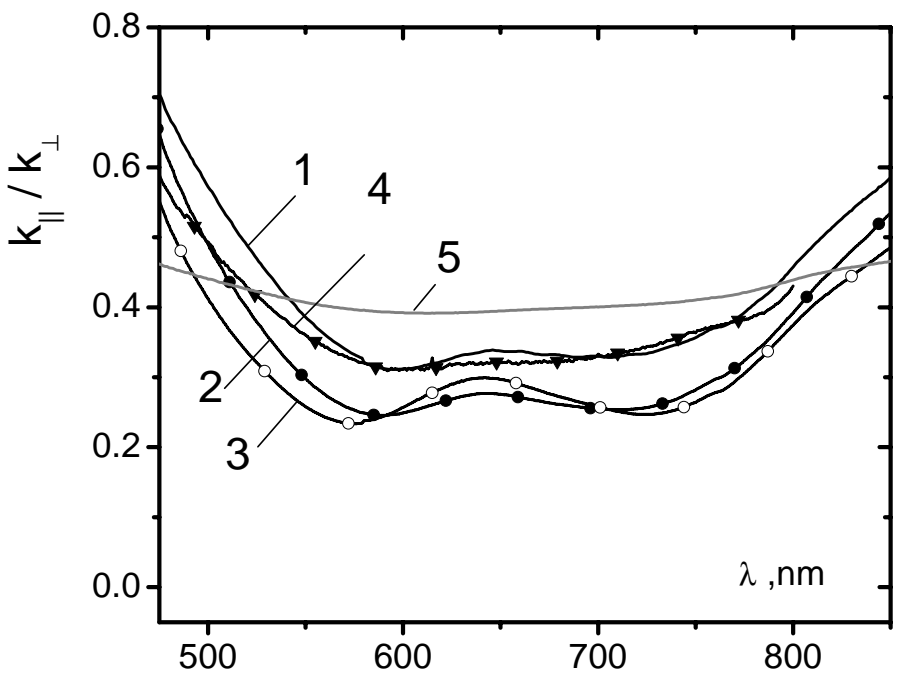

Fig. 5. The values $k_{\|} / k_{\perp}$ for Blue $2505.2 \mathrm{wt} \%$ with the ionic additives: (1) Blue 250, (2) $\left(\mathrm{C}_{4} \mathrm{H}_{9}\right)_{4} \mathrm{NI}$, (3) $\mathrm{KI}$, (4) Nal and (5) $\mathrm{Na}_{2} \mathrm{SO}_{4}$. 


\section{Scalar order parameter deduced from the dichroism}

Using the experimental values of $\mathrm{k}_{\mid} / \mathrm{k}_{\perp}$, we have calculated the scalar order parameters of the LCLC Blue 250 with the ionic additives. Consider a nematic phase formed by orientationally ordered rod-like aggregates built of plank-like molecules. The 3D nematic scalar order parameter is the coefficient of the second-order Legendre polynomial in the polynomial expansion of orientational distribution function having the form

$$
S_{3 D}=\frac{1}{2}\left(3\left\langle\cos ^{2} \theta\right\rangle-1\right)
$$

where $\theta$ is the angle between the actual direction of the long axis $\vec{A}$ of a given aggregate and the director $\vec{n}$ and $\left\langle\cos ^{2} \theta\right\rangle$ denotes a 3D thermodynamic average. The maximum possible value of $S_{3 D}$ is unity. For a light-absorbing nematic the value of $\left\langle\cos ^{2} \theta\right\rangle$ can be deduced from the dichroic ratio $N=k_{\|} / k_{\perp}$. The light absorption coefficients measured for the light polarized parallel and perpendicular to the director can be expressed respectively as

$$
\begin{aligned}
& k_{\|}=a P^{2}\left\langle\cos ^{2} \theta_{d}\right\rangle, \\
& k_{\perp}=a P^{2}\left\langle\sin ^{2} \theta_{d}\right\rangle\left\langle\sin ^{2} \varphi\right\rangle,
\end{aligned}
$$

where $a$ is a material constant, $\vec{P}$ the transition dipole moment, $\theta_{d}$ the angle between $\vec{P}$ and $\vec{n}, \varphi$ the azimuth of $\vec{P}$ with respect to the director and

$$
\left\langle\sin ^{2} \varphi\right\rangle=\frac{1}{2 \pi} \int_{0}^{2 \pi} \sin ^{2} \varphi d \varphi=\frac{1}{2} .
$$

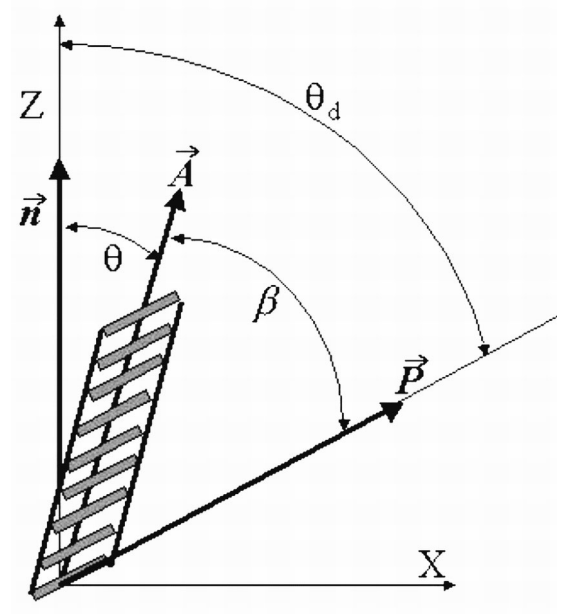

Fig.6. Schematic structure of LCLC aggregate

The dipole moment $\vec{P}$ of the constituent plank-like molecule makes a fixed angle $\varphi$ with the aggregate axis $\vec{A}$. Now the average orientation of the aggregate axis $\left\langle\cos ^{2} \theta\right\rangle$ has to be expressed in terms of the average orientation $\left\langle\cos ^{2} \theta_{d}\right\rangle$ of the transition dipole entering Eq. (2). To this end we choose the reference frame associated with the aggregate axis $\vec{A}$, in which this axis is fixed while the director and the dipole moment vector spin around. Let the $\mathrm{Z}$ axis be along $\vec{n}$ and the $\mathrm{XZ}$ plane be spanned over the vectors $\vec{A}$ and $\vec{n}$ (see Fig. 6). 
In this reference frame the dipole moment $\vec{P}$ of the constituent molecule has the azimuth $\gamma$ with respect to the $\mathrm{X}$ axis. The relation between $\theta$ and $\theta_{d}$ is now seen to be of the form

$$
\cos \theta_{d}=\cos \theta \cos \beta+\sin \theta \sin \beta \cos \gamma .
$$

Upon averaging the square of Eq. (4) over $\theta$ and $\gamma$ one obtains

$$
\left\langle\cos ^{2} \theta_{d}\right\rangle=\left\langle\cos ^{2} \theta\right\rangle \cos ^{2} \beta+\frac{1}{2}\left\langle\sin ^{2} \theta\right\rangle \sin ^{2} \beta,
$$

where $\left\langle\sin ^{2} \gamma\right\rangle=1 / 2$ (averaging over the rotation of $\mathrm{X}$ axis about $\mathrm{Z}$ axis is trivial, since Eq. (5) does not depend on $\gamma$ ). From this equation one has

$$
\left\langle\cos ^{2} \theta\right\rangle=\frac{\left\langle\cos ^{2} \theta_{d}\right\rangle-\frac{1}{2} \sin ^{2} \beta}{1-\frac{3}{2} \sin ^{2} \beta} .
$$

From Eqs. (1)-(3) and (6) the 3D scalar order parameter finally obtains the following form:

$$
S_{3 D}=\frac{N-1}{(N+2)\left(1-\frac{3}{2} \sin ^{2} \beta\right)} .
$$

In particular, for the case of $\beta=\frac{\pi}{2}$ we have

$$
S_{3 D}(\beta=\pi / 2)=\frac{1-N}{1+N / 2} .
$$

Fig. 7 demonstrates the scalar order parameter obtained for the pure Blue 250 and the Blue 250 with salt additives. The data are sorted in order of increasing scalar order parameter of the solution. It follows from the absorption anisotropy data that the addition of salts shows a tendency to increasing nematic scalar order parameter, though quantita-

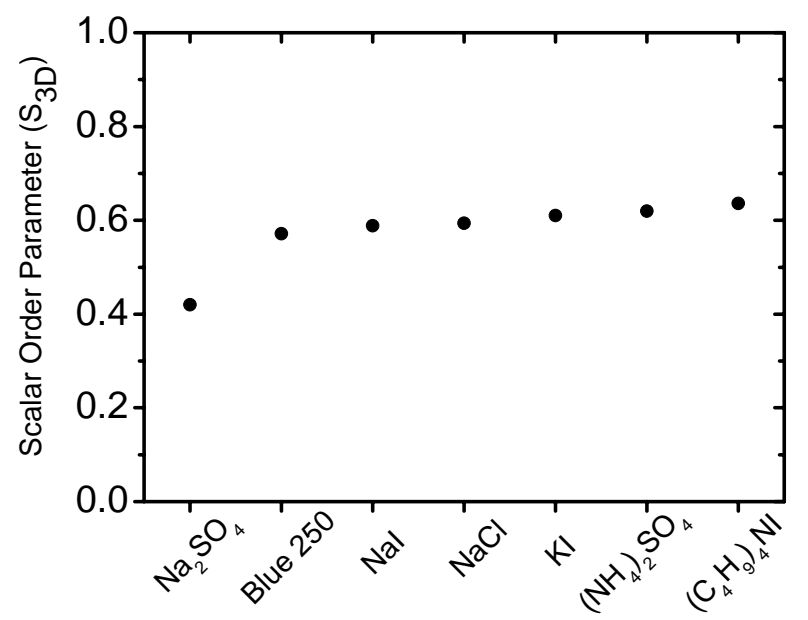

Fig. 7. Scalar order parameter for Blue $2505.2 \mathrm{wt} \%$ with the corresponding ionic additives measured at $\lambda=650 \mathrm{~nm}$. 
tively these changes are not large and range between $\mathrm{S}_{3 \mathrm{D}}=0.57$ for the pure Blue 250 and $\mathrm{S}_{3 \mathrm{D}}=0.64$ for the solution with $\left(\mathrm{C}_{4} \mathrm{H}_{9}\right)_{4} \mathrm{NI}$ added. However, the addition of $\mathrm{Na}_{2} \mathrm{SO}_{4}$ results in decreasing $\mathrm{S}_{3 \mathrm{D}}$ value down to 0.42 . This value is definitely lower than it can be expected for a stable nematic phase. We have verified carefully that this low $S_{3 D}$ value is not a result of experimental errors or a poor alignment uniformity of the sample. We have thus suggested that it is rooted in the structural organization of the chromonic aggregates. We believe that $\langle\cos \theta\rangle$ is significantly lowered due to growing side chains and branching the aggregates (i.e. transforming the aggregates from the rod shape to a Y-shaped construction), when the $\mathrm{Na}_{2} \mathrm{SO}_{4}$ salt is added.

The fact that the S parameter for the other salts hardly depends on their presence can be interpreted as an evidence that the inter-columnar interaction is not affected by the added ions, whereas the changes observed on the phase diagram allude to the above suggestion that the length of the aggregates and polydispersity of their lengths increase when the salts belonging to the first group are present. A slight increase in the order parameter supports these points.

\section{Conclusion}

We have demonstrated that the phase state and the structure of the aggregates in the water solution of Blue 250 change upon adding $\mathrm{KI},\left(\mathrm{NH}_{4}\right)_{2} \mathrm{SO}_{4}, \mathrm{NaCl}$ and $\left(\mathrm{C}_{4} \mathrm{H}_{9}\right)_{4} \mathrm{NI}$ salts, whereas the orientation scalar order parameter is not sensitive to their presence. These changes point to increase in the aggregate length and/or their polydispersity, with the intercolumnar interactions being unaffected. The $\mathrm{Na}_{2} \mathrm{SO}_{4}$ salt differs from all the other salts by lowering of the scalar order parameter, which can be explained by modification of the shape of the chromonic aggregates. These changes may be attributed to growing of side chains within the rod-like aggregates. Finally, the $\mathrm{NH}_{4} \mathrm{Cl}$ salt does not affect the phase diagram and the scalar order parameter of the Blue 250.

\section{Acknowledgement}

This work was supported by the NAS of Ukraine via the Grant No 1.4.1B/109, Fundamental Research State Fund Project UU24/018 and the Project No 0106 U000617 from the Ministry of Education and Science of Ukraine.

\section{References}

1. Sergan T, Schneider T, Kelly J and Lavrentovich O D, 2000. Polarizing alignment layers for twisted nematic cells. Liquid Crystal 27: 567-572.

2. Bobrov Y, Cobb C, Lazarev P, Bos P, Bryand D and Wonderly H, 2000. Lyotropic Thin Film Polarizers. SID, Int. Symp. Digest of Technical Papers, Long Beach, California May 16-18, XXXI: 1102-1107.

3. Wip W C, Kwok H S, Kozenkov V M and Chigrinov V G, 2001. Photopatterned ewave polarizer. Displays 22: 27-32.

4. Boiko O, Komarov O, Vasyuta R, Nazarenko V, Slominskiy Yu and Schneider T, 
2005. Nano-Architecture of Self-Assembled Monolayer and Multilayer Stacks of Lyotropic Chromonic Liquid Crystalline Dyes. Mol.Cryst.Liq.Cryst., 434: 305 - 314.

5. Boiko O P, Vasyuta R M, Nazarenko V G, Pergamenshchik V M, Nastishin Yu A and Lavrentovich O D, 2007. Polarizing Properties of Functional Optical Films Based on Lyotropic Chromonic Liquid Crystals. Mol. Cryst. Liq. Cryst. 467: 181-194.

6. Lavrentovich M, Sergan T and Kelly J, 2003. Planar and twisted lyotropic chromonic liquid crystal cells as optical compensators for twisted nematic displays. Liquid Crystal 30: 851-859.

7. Lydon J, 1998. in Handbook of Liquid Crystals, ed. by Demus D, Goodby J, Gray G W, Spiess H-W, and Vill-Wiley V, Weinheim: VCH, 2B: 981-1007.

8. Lydon J, 1998. Chromonic liquid crystal phases. Current opinion in colloid \& interface science. 3: 458-466.

9. Lydon J, 2004. Chromonic mesophases. Current opinion in colloid \& interface science. 8: 480-490.

10. Vasilevskaya A S., Generalova E V. and Sonin A S, 1989. Chromonic Mesophases. Russ. Chem. Rev. 58: 904-916.

11. Camorani P, Furier M, Kachkovskii O, Piryatinskiy Yu, Slominskiy Yu and Nazarenko V, 2001. Absorption spectra and chromonic phase in aqueous solution of perylenetetracarboxylic bisimides derivatives. Semicond. Phys., Quantum Electron. Optoelectron. 4: 229-238.

12. Nastishin Yu A, Liu H, Schneider T, Nazarenko V, Vasyuta R, Shiyanovskii S V and Lavrentovich O D, 2005. Optical characterization of the nematic lyotropic chromonic liquid crystals: light absorption, birefringence, and scalar order parameter. Phys. Rev. E 72: 041711:1-14.

13. Vasyuta R, Boiko O, Piryatinskiy Yu, Nazarenko V and Kachkovsky A, 2005. Absorption and Fluorescence Spectra of Aqueous Solutions of Disodium Chromoglycate. Mol. Cryst. Liq. Cryst., 426: 117-127.

14. Nastishin Yu A., Liu H, Shiyanovskii S V, Lavrentovich O D, Kostko A F. and Anisimov M A, 2005. Pretransitional fluctuations in the isotropic phase of a lyotropic chromonic liquid crystal. Phys. Rev. E 70: 051706:1-9.

15. Horowitz V R, Janowitz L A, Modic A L, Heiney P A and Collings P J, 2005. Aggregation behavior and chromonic liquid crystal properties of an anionic monoazo dye Phys. Rev. E 72: 041710:1-10.

16. Harrison W J, Mateer D L and Tiddy G T, 1996. Liquid-Crystalline J-Aggregates Formed by Aqueous Ionic Cyanine Dyes. J. Phys. Chem. 100: 2310-2314.

17. Andrei F Kostko, Bani H Cipriano, Olga A Pinchuk, Lior Ziserman, Mikhail A Anisimov, Dganit Danino and Srinivasa R Raghavan, 2005. Salt Effects on the Phase Behavior, Structure, and Rheology of Chromonic Liquid Crystals. J. Phys. Chem. B 109: 19126-19133.

18. Lee H and Labes M M, 1982. Lyotropic Cholesteric and Nematic Phases of Disodium Cromoglycate in Magnetic Fields. Mol. Cryst. Liq. Cryst. 84: 137-157. 
19. Yu L J, Saupe A, 1982. Deuteron Resonance of $\mathrm{D}_{2} \mathrm{O}$ of Nematic Disodium Cromoglycate-Water Systems Mol. Cryst. Liq. Cryst. 80: 129-134.

20. Perahia D, Goldfarb D, and Luz Z, 1984. Sodium-23 NMR in the lyomesophases of disodiumcromoglicate. Mol. Cryst. Liq. Cryst. 108: 107-123.

21. Mundy K, Sleep J C and Lydon J E, 1995. The intercalation of ethidium bromide in the chromonic lyotropic phases of drugs and nucleic acids. Liq. Cryst. 19: 107-112.

22. Park H S, Tortora L, Vasyuta R M, Golovin A B, Augustin E, Finotello D and Lavrentovich O D, 2007. Lyotropic Chromonic Liquid Crystals: Effects of Additives and Optical Applications. IMID '07, Digest of Technical Papers, 307-310.

23. Hartshorne N H and Woodard G D, 1973. Mesomorphism in the system disodiumchromoglicate - water. Mol. Cryst. Liq. Cryst. 23: 343-368.

24. Taylor M P and Herzfeld J, 1991. Shape anisotropy and ordered phases in reversibly assembling lyotropic systems. Phys. Rev. A 43: 1892-1905.

25. Barrat J L and Hansen J P, Basic Concepts for Simple and Complex Liquids. New York: Cambridge University Press, (2003).

26. MacKintosh FC, Safran S A and Pincus P A, 1990. Self-Assembly of Linear Aggregates: the Effect of Electrostatics on Growth. J. Phys.: Condens. Matter 2: SA359-364.

27. Speranza A and Sollich P J, 2002. Onsager theory for isotropic nematic phase equilibria of length polydisperse hard rods. Chem. Phys. 117: 5421-5436.

28. Wensink H H and Vroege G J, 2003. Isotropic-nematic phase behavior of lengthpolydisperse hard rods. J. Chem. Phys. 119: 6868-6882. 